\title{
CD44, Sonic Hedgehog, and Gli1 Expression Are Prognostic Biomarkers in Gastric Cancer Patients after Radical Resection
}

\author{
Chen Jian-Hui, ${ }^{1,2}$ Zhai Er-Tao, ${ }^{1,2}$ Chen Si-Le, ${ }^{1,2}$ Wu Hui, ${ }^{1,2}$ Wu Kai-Ming, ${ }^{1,2}$ \\ Zhang Xin-Hua, ${ }^{1,2}$ Chen Chuang-Qi, ${ }^{1,2}$ Cai Shi-Rong, ${ }^{1,2}$ and He Yu-Long, \\ ${ }^{1}$ Division of Gastrointestinal Surgery Center, The First Affiliated Hospital of Sun Yat-sen University, Guangzhou 510080, China \\ ${ }^{2}$ Gastric Cancer Center, Sun Yat-sen University, Guangzhou 510080, China
}

Correspondence should be addressed to Cai Shi-Rong; drcaishirong@163.com

Received 11 September 2015; Revised 25 October 2015; Accepted 16 November 2015

Academic Editor: Mitsuro Kanda

Copyright (c) 2016 Chen Jian-Hui et al. This is an open access article distributed under the Creative Commons Attribution License, which permits unrestricted use, distribution, and reproduction in any medium, provided the original work is properly cited.

\begin{abstract}
Aim. CD44 and Sonic Hedgehog (Shh) signaling are important for gastric cancer (GC). However, the clinical impact, survival, and recurrence outcome of CD44, Shh, and Glil expressions in GC patients following radical resection have not been elucidated. Patients and Methods. CD44, Shh, and Glil protein levels were quantified by immunohistochemistry (IHC). The association between CD44, Shh, and Glil expression and clinicopathological features or prognosis of GC patients was determined. The biomarker risk score was calculated by the IHC staining score of CD44, Shh, and Glil protein. Results. The IHC positive staining of CD44, Shh, and Glil proteins was correlated with larger tumour size, worse gross type and histological type, and advanced TNM stage, which also predicted shorter overall survival (OS) and disease-free survival (DFS) after radical resection. Multivariate analysis indicated the Glil protein and Glil, CD44 proteins were predictive biomarkers for OS and DFS, respectively. If biomarker risk score was taken into analysis, it was the independent prognostic factor for OS and DFS. Conclusions. CD44 and Shh signaling are important biomarkers for tumour aggressiveness, survival, and recurrence in GC.
\end{abstract}

\section{Introduction}

Due to an increased early detection rate and therapeutic advancements, the survival of gastric cancer (GC) patients has improved in the past 3 decades worldwide. However, GC remains the second leading cause of cancer death in China [1], mainly because of the disappointing early detection rate in China, early tumour recurrence, and high chemotherapy resistance. Hence, it is essential for gastroenterologists to identify effective biomarkers for evaluating the early detection of GC, which may also be targets for novel therapies for this deadly disease.

Cancer stem-like cells (CSCs) are defined as rare cells in malignant tumours with the ability to self-renew and to differentiate into various heterogeneous cancer cell lineages [2]. Abnormal gene expression in CSCs might be responsible for the acquisition of various genetic and epigenetic events and may play a critical role in tumour initiation, maintenance, progression, lymphatic involvement, distant metastasis, and chemoradiotherapy resistance [3]. Therefore,
CSCs are considered promising tumour-specific biomarkers with potential clinical application. CD44, widely accepted as a CSCs marker for gastric cancer in many studies [4-6], is involved in cell-cell adhesion, cell-matrix interactions, and tumour metastasis [4]. However, most studies exploring the role of CD44 protein in gastric cancer included patients that received either radical resection or palliative surgery, which introduced bias into the studies. Hence, it is necessary to reevaluate the relationship between CD44 expression and clinicopathological features and long-term survival of GC patients who received radical resection. The activation of the Sonic Hedgehog (Shh) pathway affects numerous human stem cell markers in prostate [7], breast [8], and pancreatic [9] cancer. Several studies have demonstrated that increased CD44 expression activates several signalling pathways related to cancer progression and metastasis, including Shh pathway [10]. Song et al. [10] demonstrated that the Shh pathway is essential for maintenance of human gastric cancer CSCs in vitro. However, the clinical impact and interaction between the Shh pathway and CD44 expression in gastric cancer 
patients are still uncertain. Here, we aimed to find out the correlations between CD44, Glil, and Shh expression and clinicopathological features, long-term survival, and recurrence.

\section{Methods}

2.1. Ethic Statement. The study was approved by the Institutional Review Board of the 1st Affiliated Hospital of Sun Yatsen University and informed consent was obtained according to institutional regulations.

2.2. Clinical Samples. A total of 101 primary gastric cancer tissues were obtained at the 1st Affiliated Hospital of Sun Yat-sen University, Guangzhou, China, between January 2006 and June 2006. Patients who underwent radical gastrectomy were included, while patients who received neoadjuvant chemotherapy or chemoradiotherapy were excluded from the study. Clinicopathological parameters evaluated included age, gender, tumour location, tumour size, gross tumour type, tumour histological type, depth of invasion, lymph node involvement, distant metastasis, and TNM stage. Tumour gross types were classified as either infiltrating or noninfiltrating. Tumour histological types were classified as either well differentiated (well and moderately differentiated adenocarcinomas) or undifferentiated (poorly differentiated adenocarcinomas, signet ring cell carcinomas, and mucinous adenocarcinomas). Depth of tumour invasion, lymph node involvement, and distant metastasis were assessed according to the 7th edition of Union for International Cancer Control/American Joint Committee on Cancer (UICC/AJCC) guidelines. The potential radical resection gastric cancer patients received gastrectomy and D2 lymphadenectomy. The patients received postoperative chemotherapy using epirubicin, cisplatin, and 5-fluorouracil regimen as indicated by the concurrent UICC/AJCC guidelines. In addition, 20 human adjacent normal gastric tissues were obtained. Chronic atrophic gastritis, ulcer, and erosion were not detected microscopically in the adjacent gastric tissue samples.

\subsection{Immunohistochemical Staining. Formalin fixed paraffin} embedded human gastric cancer specimens were prepared according to the classical methods. The sections $(5 \mu \mathrm{m}$ thickness) were treated with protein-blocking solution for $30 \mathrm{~min}$ at temperature before being incubated with primary antibodies against human CD44 (mouse monoclonal diluted $1: 50$ ), Shh (rabbit polyclonal diluted 1:100), and Glil (mouse polyclonal diluted $1: 100$ ) overnight at $4^{\circ} \mathrm{C}$. All antibodies were obtained from Novus Biologicals (USA). Following incubation with the appropriate peroxidase-conjugated secondary antibody, the samples were treated with diaminobenzidine and counterstained with hematoxylin. Using brightfield microscopy, the percentage of positive cancer cells and the staining intensity was quantified independently by 2 pathologists. The mean percentage of positive tumour cells was quantified in at least 5 fields at 400x magnification and classified into one of the following 5 grades: $0 \quad(<5 \%$ of cells had positive staining), 1 (5-25\% of cells had positive staining), 2 (26-50\% of cells had positive staining), 3 (51$75 \%$ of cells had positive staining), and 4 ( $>75 \%$ of cells had positive staining). The staining intensity of CD44, Shh, and Glil was scored as follows: 0 (no staining), 1 (light brown), 2 (brown), and 3 (dark brown). The percentage score and staining intensity score were multiplied to get the final staining score for each tumour specimen. The overall staining scoring system could be categorised into 2 groups: negative (0-4), positive (5-12). We defined the positive IHC staining of biomarker as biomarker overexpression.

2.4. Statistical Analysis. The biomarker risk score for gastric cancer in this study was the sum of the IHC score of CD44, Shh, and Glil proteins (positive: score 1, negative: score 0), and the patients were divided into four groups according to biomarker risk scores (groups 1-4: score 0-3). Continuous variables are presented as the mean \pm SEM and categorical variables are presented as percentages (\%). The two-tailed Chi-square test and Fisher's exact test for categorical variables were performed to determine statistical significance of the associations between clinicopathological parameters and the level of CD44, Shh, and Glil expression. Overall survival and disease-free survival rates were calculated according to the Kaplan-Meier method and were compared by log-rank tests. Cox proportional hazard models were performed for both univariate and multivariate analysis to determine prognostic significance. Spearman's rank order correlation was used to determine the correlations between the expressions of CD44, Shh, and Glil. A $P$ value of less than 0.05 was considered as statistically significant. SPSS 16.0 software (version 17.0, SPSS Inc., Chicago, IL) was used for all statistical analyses.

\section{Results}

3.1. Correlations between CD44, Shh, and Gli1 Expression and Clinicopathological Characteristics of Gastric Cancer. To investigate the role of the tumour stem cell biomarker CD44 and Shh signaling pathway in GC tumour, we evaluated the levels of CD44, Shh, and Glil protein in tumour tissues using immunohistochemistry (IHC) (Figure 1) and the positive stainings of Glil, Shh, and CD44 protein were mainly localized in the nucleus, cytoplasm, and cell membrane, respectively. We found that 57.8\% (59/101), 71.3\% (72/101), and $57.8 \%$ (59/101) GC tumour specimens stained positively for CD44, Shh, and Glil protein, respectively. To further investigate the effect of CD44, Shh, and Glil in gastric cancer progression, we analysed the correlations between the level of CD44, Shh, and Glil protein and clinicopathological characteristics of GC. There were no statistically significant correlations between CD44, Shh, and Glil expression levels and age, gender, or tumour location (Table 1). Overexpression of CD44, Shh, and Glil protein was significantly associated with larger tumour size, aggressive gross type, and less differentiated tumour histological type, all of which were clinicopathological features associated with a high metastatic potential. Tumours with high CD44, Shh, and Glil expression had more cases of advanced tumour invasion, an increased 

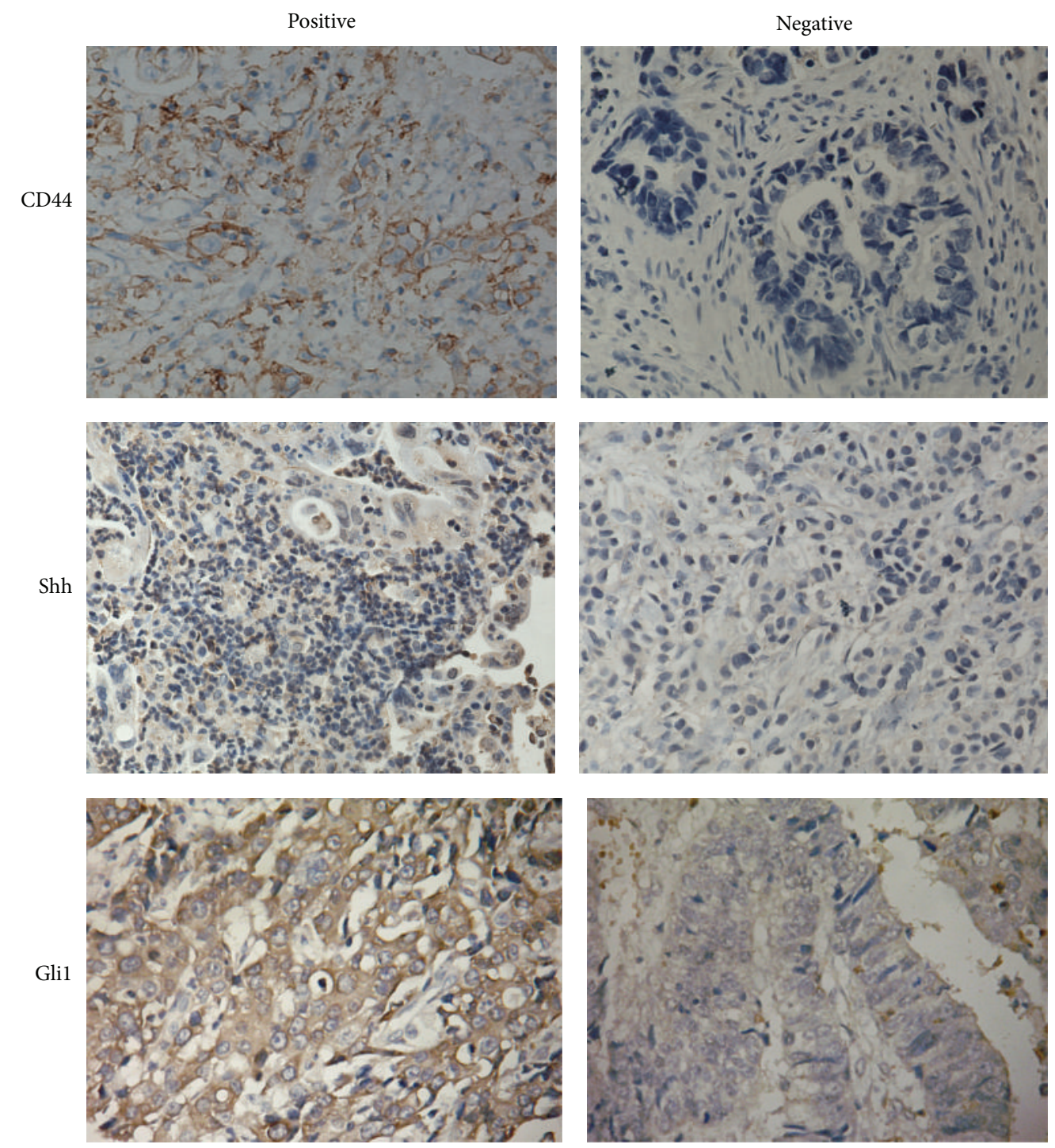

FIGURE 1: Immunohistochemical expressions of CD44, Shh, and Glil markers.

likelihood of lymph node metastasis, advanced TNM stage (Table 1).

3.2. The Overexpression of CD44, Shh, and Gli1 Proteins Indicated Poor Clinical Outcome. Using Kaplan-Meier analysis and the log-rank test, we find that gastric cancer patients with CD44-positive staining had poorer overall survival. $73.8 \%$ of patients with CD44-negative tumours survived 5 years compared to only $27.1 \%$ of patients with CD44-positive tumours (Figure $2(\mathrm{a}))(P<0.001)$. A similar result was observed when CD44 expression status and recurrence-free survival time were compared. The recurrence-free survival time of patients with CD44-positive tumours was lower than that of patients with CD44-negative tumours (39.0\% versus $79.5 \%$, resp., $P=0.001$ ) (Figure 2(b)). Similarly, cases with Shh and Glil positive staining had poorer overall survival (Shh: $33.3 \%$ versus $79.3 \%, P<0.001$; Glil: $21.3 \%$ versus $85.0 \%$, $P<0.001)$ and recurrence-free survival (Shh: $44.6 \%$ versus
84.9\%, $P<0.001$; Gli1: $35.8 \%$ versus $86.5 \%, P<0.001)$ (Figures 2(c)-2(f)).

In accordance with these results, univariate Cox regression analysis also revealed that CD44, Shh, and Glil status were associated with the prognosis of gastric cancer in our study (Table 2). Rather than CD44 and Shh expression levels, only TNM staging and Glil expression level were independent prognostic factors for overall survival of patients with GC in this study (Table 2). Similar to the results of prognostic analysis for overall survival, CD44, Shh, and Gli1 status also affected the recurrence of gastric cancer in our study (Table 3). The multivariate analysis revealed that, other than TNM stage and nodal classification, Glil status was the independent factor for recurrence-free survival in our study (Table 3).

3.3. The Correlation of CD44 Expression with the Shh Signalling Pathway in Gastric Cancer. The Shh signalling pathway regulates tumour development via cell proliferation and 


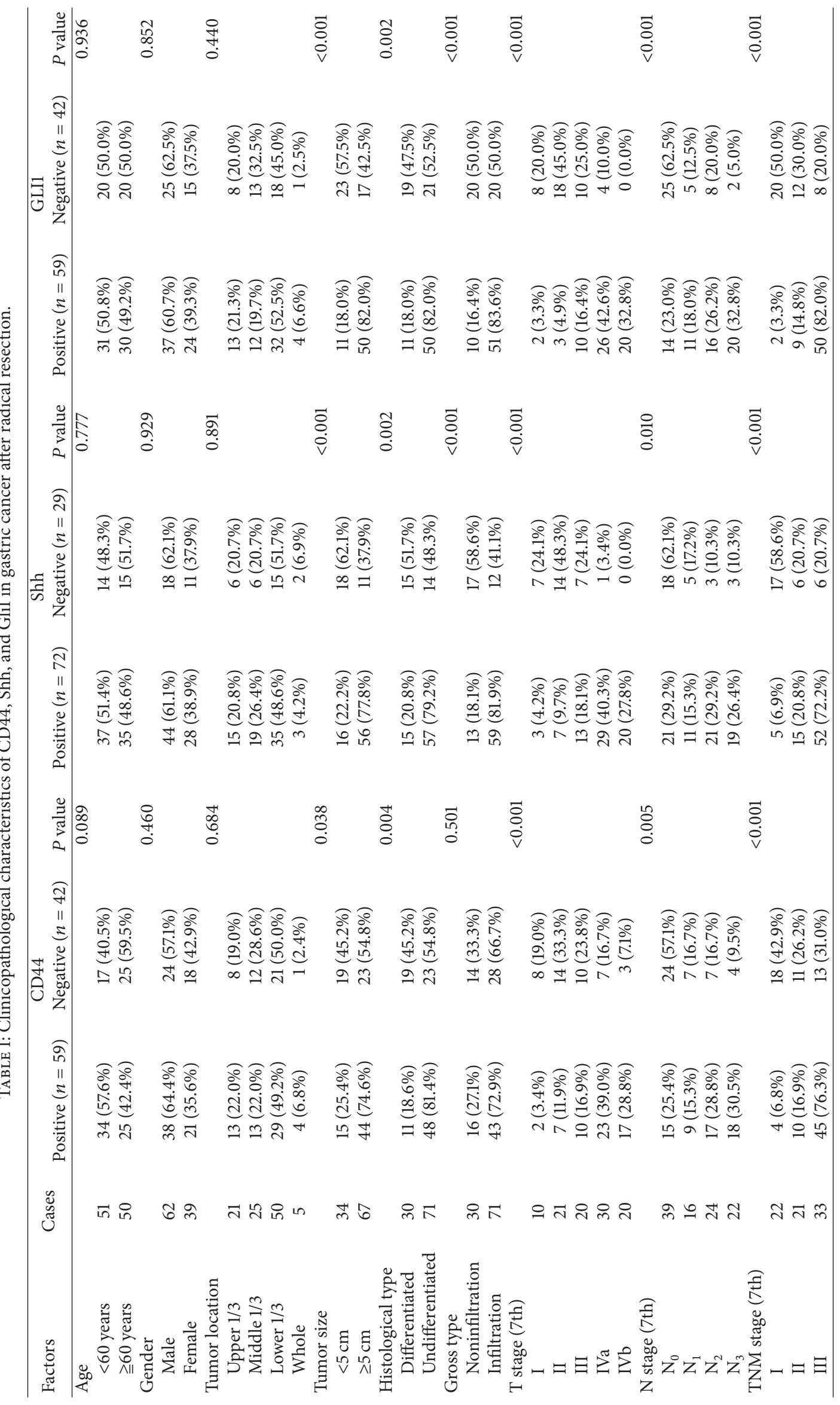




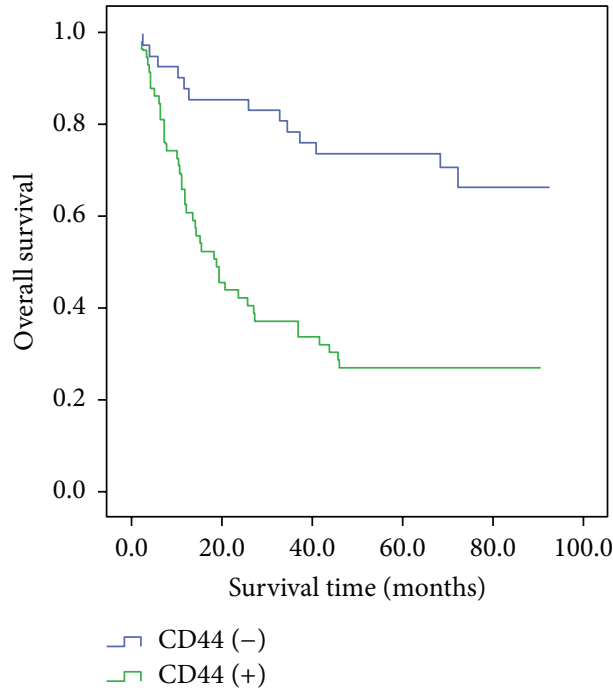

(a)

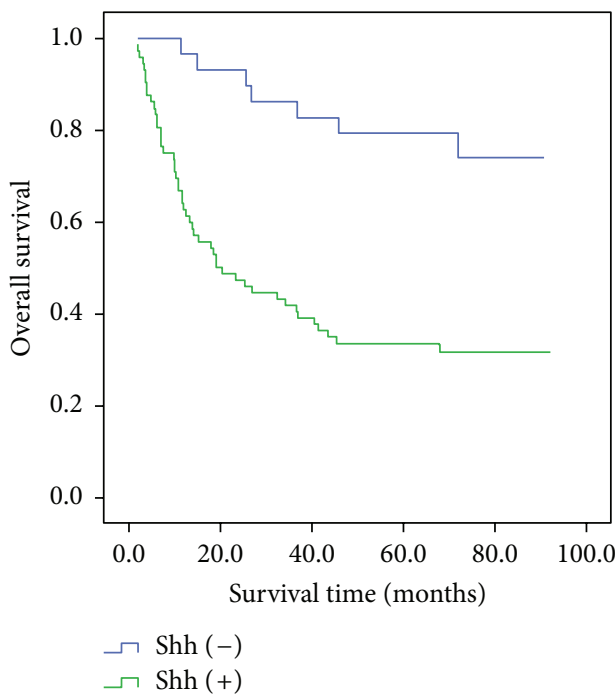

(c)

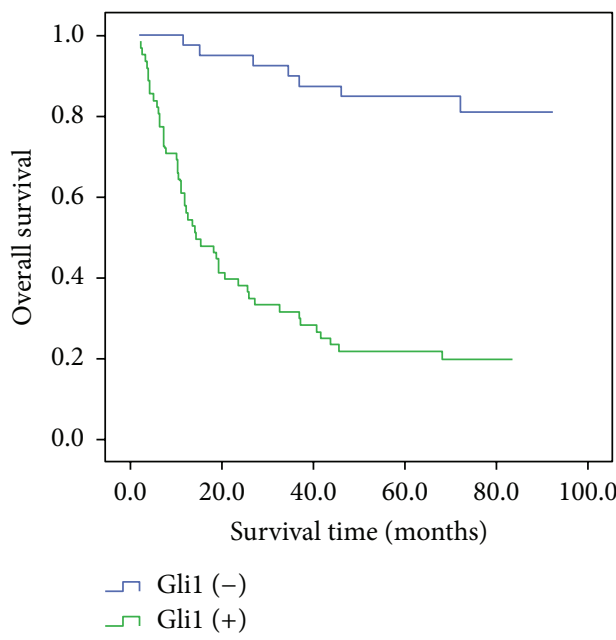

(e)

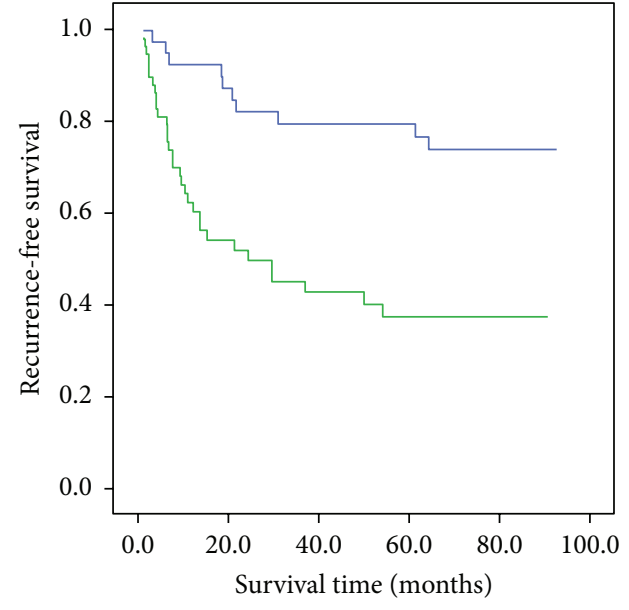

$\neg \mathrm{CD} 44(-)$

$\neg \mathrm{CD} 44(+)$

(b)

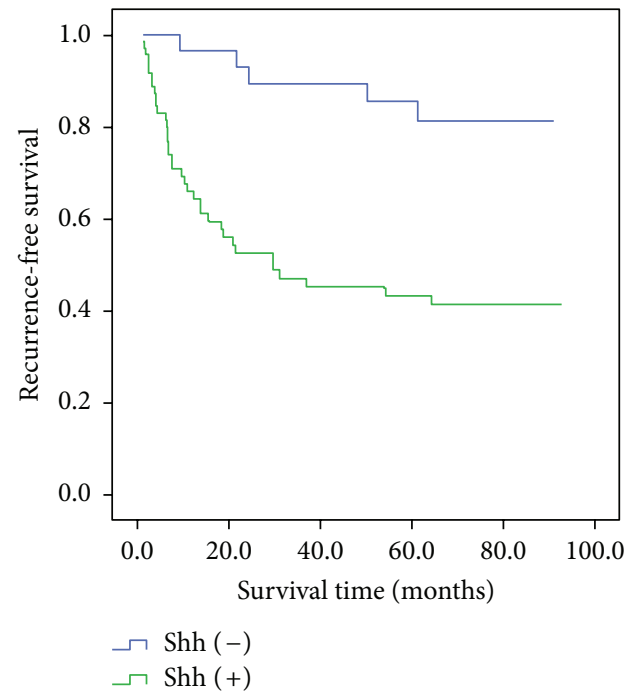

(d)

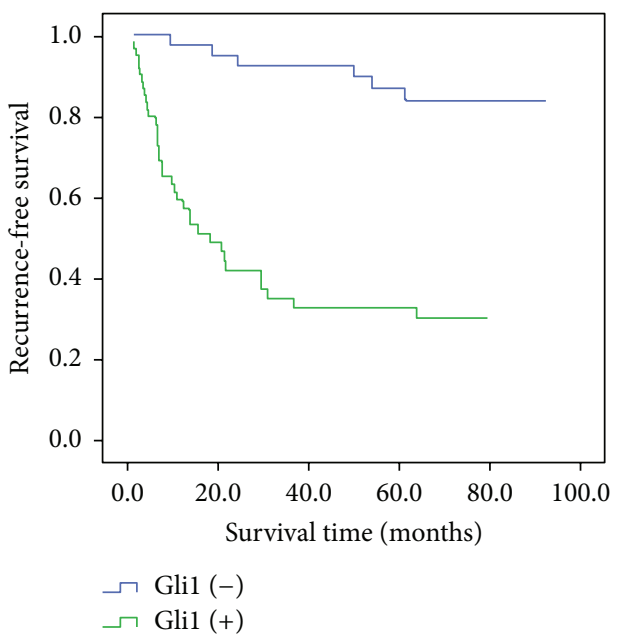

(f)

FIGURE 2: Prognostic impact of CD44, Shh, and Glil markers. (a) CD44 and overall survival, (b) CD44 and recurrence-free survival, (c) Shh and overall survival, (d) Shh and recurrence-free survival, (e) Glil and overall survival, and (f) Glil and recurrence-free survival. 
TABLE 2: Univariate and multivariate analysis for overall survival in gastric cancer after radical resection.

\begin{tabular}{|c|c|c|c|c|c|c|c|c|}
\hline \multirow{2}{*}{ Factors } & \multicolumn{4}{|c|}{ Univariate regression analysis } & \multicolumn{4}{|c|}{ Multivariate regression analysis } \\
\hline & $\chi^{2}$ value & OR & $95 \%$ CI & $P$ value & $\chi^{2}$ value & OR & $95 \%$ CI & $P$ value \\
\hline Age & 0.001 & 0.995 & $0.589-1.679$ & 0.984 & & & & \\
\hline Gender & 0.076 & 0.927 & $0.540-1.592$ & 0.783 & & & & \\
\hline Tumor location & 0.052 & 0.966 & $0.713-1.307$ & 0.820 & & & & \\
\hline Tumor size & 10.301 & 2.957 & $1.525-5.733$ & 0.001 & & & & \\
\hline Histological type & 7.097 & 2.457 & $1.268-4.760$ & 0.008 & & & & \\
\hline Gross type & 5.811 & 2.252 & $1.164-4.357$ & 0.016 & & & & \\
\hline T stage & 11.943 & 3.370 & $1.692-6.713$ & 0.001 & & & & \\
\hline $\mathrm{N}$ stage & 5.334 & 2.473 & $1.147-5.333$ & 0.021 & & & & \\
\hline TNM stage & 21.978 & 3.070 & $1.921-4.906$ & $<0.001$ & 11.856 & 1.346 & $1.137-1.594$ & 0.001 \\
\hline CD44 expression & 16.049 & 3.589 & $1.921-6.706$ & $<0.001$ & & & & \\
\hline Shh expression & 13.707 & 4.490 & $2.028-9.945$ & $<0.001$ & & & & \\
\hline Glil expression & 28.800 & 8.927 & $4.013-19.858$ & $<0.001$ & 9.970 & 4.247 & $1.731-10.423$ & 0.002 \\
\hline
\end{tabular}

TABLE 3: Univariate and multivariate analysis for disease-free survival in gastric cancer after radical resection.

\begin{tabular}{|c|c|c|c|c|c|c|c|c|}
\hline \multirow{2}{*}{ Factors } & \multicolumn{4}{|c|}{ Univariate regression analysis } & \multicolumn{4}{|c|}{ Multivariate regression analysis } \\
\hline & $\chi^{2}$ value & OR & $95 \%$ CI & $P$ value & $\chi^{2}$ value & OR & $95 \%$ CI & $P$ value \\
\hline Age & 0.461 & - & - & 0.497 & & & & \\
\hline Gender & 0.323 & - & - & 0.570 & & & & \\
\hline Tumor location & 0.706 & - & - & 0.401 & & & & \\
\hline Tumor size & 8.632 & 3.187 & $1.471-6.904$ & 0.003 & & & & \\
\hline Histological type & 2.391 & - & - & 0.122 & & & & \\
\hline Gross type & 4.816 & 2.372 & $1.097-5.130$ & 0.028 & & & & \\
\hline T stage & 20.271 & 1.912 & $1.442-2.535$ & $<0.001$ & & & & \\
\hline $\mathrm{N}$ stage & 20.429 & 1.841 & $1.413-2.398$ & $<0.001$ & 4.368 & 1.334 & $1.018-1.747$ & 0.037 \\
\hline TNM stage & 27.076 & 2.663 & $1.841-3.851$ & $<0.001$ & 7.473 & 1.940 & $1.206-3.121$ & 0.006 \\
\hline CD44 expression & 11.981 & 3.545 & $1.731-7.258$ & 0.001 & & & & \\
\hline Shh expression & 10.853 & 4.836 & $1.893-12.352$ & 0.001 & & & & \\
\hline Glil expression & 21.233 & 7.806 & $3.257-18.707$ & $<0.001$ & 6.387 & 3.403 & $1.316-8.796$ & 0.011 \\
\hline
\end{tabular}

is involved in the progression and metastasis of a wide variety of human cancers. Hence, abnormal activation of the Shh pathway could be essential for maintenance and regulation of cancer stem-like cells in human gastric cancer. Using immunohistochemistry, we found that CD44 protein levels were correlated with those of both Shh $(r=0.385, P<0.001)$ and Glil ( $r=0.219, P=0.028)$.

\subsection{Survival Impact of Biomarker Risk Score for Gastric} Cancer. We defined the positive staining of CD44, Shh, and Glil proteins as score 1, and the patients were divided into four groups according to biomarker risk scores. There were prognostic differences of overall survival and recurrencefree survival among four groups (Figures 3(a) and 3(b)), and the 5-year overall survival rates and recurrence-free survival rates of biomarker risk score of $0,1,2$, and 3 were $93.8 \%$, $72.7 \%, 57.9 \%$, and $11.4 \%$ and $100.0 \%, 75.6 \%, 61.1 \%$, and $27.3 \%$, respectively.

The biomarker risk score also had prognostic impact for overall survival $\left(\chi^{2}, 34.163\right.$; relative risk (RR), 2.766; 95\% confidence interval (CI), 1.966-3.890; $P<0.001$ ) and recurrence-free survival $\left(\chi^{2}, 25.616\right.$; RR, 2.727; 95\% CI, 1.849-4.022; $P<0.001)$. Moreover, if biomarker risk score was taken into multivariate Cox regression analysis, rather than CD44, Shh, and Glil expression, biomarker risk score $\left(\chi^{2}, 11.744 ; \mathrm{RR}, 1.999 ; 95 \% \mathrm{CI}, 1.345-2.972 ; P=0.001\right)$, and TNM stage were independent prognostic factors for overall survival, and biomarker risk score $\left(\chi^{2}, 7.183\right.$; RR, 1.848; 95\% CI, 1.179-2.895; $P=0.007)$, TNM stage, and nodal classification were independent prognostic factors for recurrence-free survival in our study.

\section{Discussion}

The CD44 gene, located on chromosome 11p12-13, has various isoforms consisting of at least 19 exons. The CD44 protein is a class I transmembrane glycoprotein and is a major component of the extracellular matrix that regulates the function of cell-cell and cell-tissue adhesion. Moreover, the CD44 protein has been identified as a biomarker of side population cells [11] or cancer stem-like cells [12] in the gastric cell lines MKN-45, MKN-74, NCI-N87, and BGC-823. 


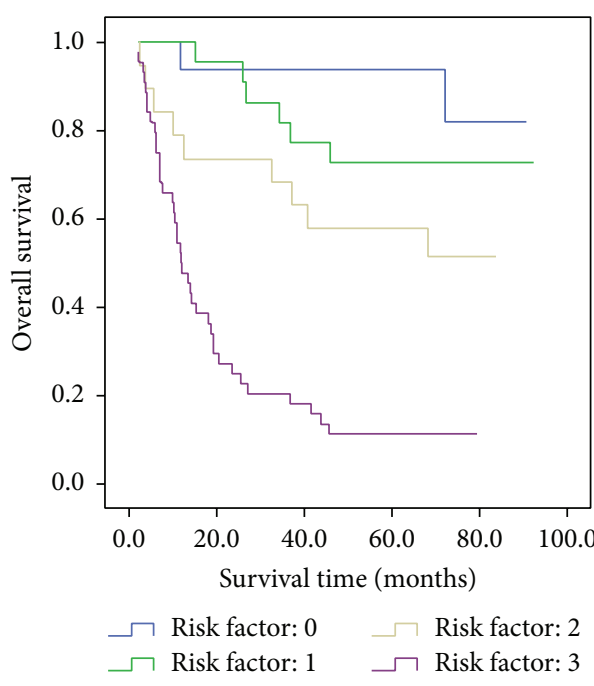

(a)

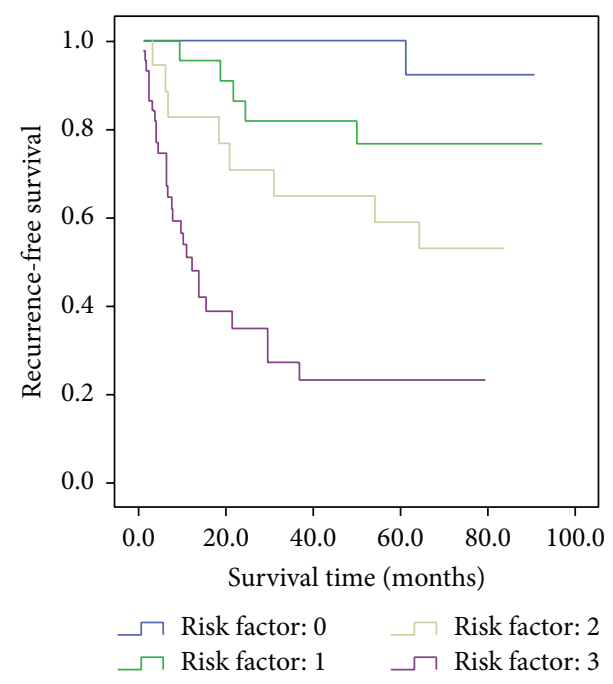

(b)

FIGURE 3: Prognostic impact of biomarker risk score system. (a) Overall survival. (b) Recurrence-free survival.

Hence, CD44 may be involved in several malignant biological processes, such as tumour initiation, development, and metastasis. As one of the most important signalling pathways, Shh has been implicated in the regulation of gastric cancer cell proliferation, migration, invasion, stem cell maintenance, and lymphangiogenesis. CD44 is required for Shh signalling pathway activation in various types of cancer, including ovarian [13], pancreatic [14], and prostate cancers [15]. Most studies have confirmed an interaction between CD44 and the Shh pathway in vivo. In contrast, Nanashima et al. [16] found no significant correlation between the expressions of Glil and CD44 in intrahepatic cholangiocarcinoma. There are very few studies in the literature evaluating the interaction between the Shh pathway and CD44 in gastric cancer cells. Song et al. [10] demonstrated that the Shh pathway was important for maintenance of cancer stem-like abilities in human gastric cancer cells. Yu et al. [17] found that overexpression of Shh signalling pathway genes was accompanied by an increase in CD44-positive cells in the MKN45 gastric cancer cell line. A similar result has been reported for breast cancer cells [18]. However, to the best of our knowledge, the correlation of CD44, Shh, and Glil in gastric cancer and their clinicopathological significance have not been reported in the literature. This is the first report revealing a positive relationship between CD44 expression and the levels of 2 important members of Hedgehog signalling pathway in vivo, suggesting that the interaction of CD44 and the Shh pathway may be involved in primary gastric cancer tumourigenesis, progression, and metastasis.

Most studies confirm that high CD44 [19], Shh [20], and Gli1 [21] expression is significantly associated with poorer clinicopathological parameters and worse overall survival in gastric cancer. It was worthy of note that most studies did not distinguish between patients who underwent radical resection and those receiving palliative surgery, which have significant differences in clinicopathological features and prognosis, when assessing the association of CD44, Shh, and Glil protein levels in GC. This study is the first to explore CD44, Shh, and Glil expression only in patients who underwent radical resection. Similar to studies that included both patients who underwent radical resection and palliative surgery, we also found an association between high CD44, Shh, and Glil expression and clinicopathological characteristics indicative of increased malignant potential, such as gross type, tumour differentiation, tumour invasion, and lymph node metastasis.

The clinical usefulness of CD44 expression to predict recurrence in GC is controversial. Hirata et al. [22] reported that expression of $\mathrm{CD} 44$ variant 9, an isoform of $\mathrm{CD} 44$, could predict recurrence in early gastric cancer. In contrast, Yong et al. found that the expression of CD44 was not associated with recurrence of gastric cancer [23]. The different proportion of patients receiving radical resection versus palliative surgery may have contributed to the different conclusions reached in these two studies. No previous studies have clarified the association between CD44 overexpression and tumour relapse or long-term survival only in gastric cancer patients who received radical resection. Moreover, this is the first study to demonstrate that patients with CD44negative tumours have better overall survival and lower recurrence rate than patients with CD44-positive tumours after radical surgery. Similarly, it is also the first time to evaluate the overexpression of Shh and Glil proteins can predict worse survival outcome and early recurrence in gastric cancer.

To assess the aggressiveness of CD44, Shh, and Glil for gastric cancer, we established biomarker risk score system to evaluate the prognostic importance. The biomarker risk score system can discriminate survival differences of overall survival and recurrence-free survival and show the highest prognostic value from the multivariate Cox regression analysis result. This may partially explain why CD44 and Shh signaling pathway signatures are useful biomarkers for aggressive tumour behaviour in gastric cancer. 
In summary, the cancer stem cell biomarker CD44 and Shh signaling pathway signatures can be used as novel diagnostic and therapeutic tools. It is necessary to further elucidate the mechanisms of aberrant Shh, Glil expression and the overexpression of CSCs markers in gastric cancer.

\section{Ethical Approval}

All procedures performed in studies involving human participants were in accordance with the ethical standards of the institutional and/or national research committee and with the 1964 Helsinki declaration and its later amendments or comparable ethical standards.

\section{Conflict of Interests}

The authors declare that they have no conflict of interests.

\section{Authors' Contribution}

Chen Jian-Hui and Zhai Er-Tao contributed equally to this work.

\section{Acknowledgments}

This study was supported by research grants from Financial Disclosure information. This study was supported by research grants from the National Nature Science Foundation of China (no. 8137234, no. 81272637, and no. 81372341) and Guangdong Province Natural Science Fund of China (no. 2014A030310111).

\section{References}

[1] J. Ferlay, H.-R. Shin, F. Bray, D. Forman, C. Mathers, and D. M. Parkin, "Estimates of worldwide burden of cancer in 2008: GLOBOCAN 2008," International Journal of Cancer, vol. 127, no. 12, pp. 2893-2917, 2010.

[2] P. Scaffidi and T. Misteli, "In vitro generation of human cells with cancer stem cell properties," Nature Cell Biology, vol. 13, no. 9, pp. 1051-1063, 2011.

[3] M. L. O’Connor, D. Xiang, S. Shigdar et al., "Cancer stem cells: a contentious hypothesis now moving forward," Cancer Letters, vol. 344, no. 2, pp. 180-187, 2014.

[4] W. Y. Jung, Y. Kang, H. Lee et al., "Expression of moesin and CD44 is associated with poor prognosis in gastric adenocarcinoma," Histopathology, vol. 63, no. 4, pp. 474-481, 2013.

[5] K. Ghaffarzadehgan, M. Jafarzadeh, H. R. Raziee et al., "Expression of cell adhesion molecule CD44 in gastric adenocarcinoma and its prognostic importance," World Journal of Gastroenterology, vol. 14, no. 41, pp. 6376-6381, 2008.

[6] C. B. da Cunha, C. Oliveira, X. Wen et al., "De novo expression of CD44 variants in sporadic and hereditary gastric cancer," Laboratory Investigation, vol. 90, no. 11, pp. 1604-1614, 2010.

[7] H.-H. Chang, B.-Y. Chen, C.-Y. Wu et al., "Hedgehog overexpression leads to the formation of prostate cancer stem cells with metastatic property irrespective of androgen receptor expression in the mouse model," Journal of Biomedical Science, vol. 18, no. 1, article 6, 2011.
[8] S. Gangopadhyay, A. Nandy, P. Hor et al., "A novel therapeutic target," Clinical Breast Cancer, vol. 13, no. 1, pp. 7-15, 2013.

[9] F. C. Kelleher, "Hedgehog signaling and therapeutics in pancreatic cancer," Carcinogenesis, vol. 32, no. 4, pp. 445-451, 2011.

[10] Z. Song, W. Yue, B. Wei et al., "Sonic hedgehog pathway is essential for maintenance of cancer stem-like cells in human gastric cancer," PLoS ONE, vol. 6, no. 3, Article ID e17687, 2011.

[11] H. Zhang, H. Xi, A. Cai et al., "Not all side population cells contain cancer stem-like cells in human gastric cancer cell lines," Digestive Diseases and Sciences, vol. 58, no. 1, pp. 132-139, 2013.

[12] S. Takaishi, T. Okumura, S. Tu et al., "Identification of gastric cancer stem cells using the cell surface marker CD44," STEM CELLS, vol. 27, no. 5, pp. 1006-1020, 2009.

[13] A. D. Steg, K. S. Bevis, A. A. Katre et al., "Stem cell pathways contribute to clinical chemoresistance in ovarian cancer," Clinical Cancer Research, vol. 18, no. 3, pp. 869-881, 2012.

[14] J. Yao, Y. An, J.-S. Wie et al., "Cyclopamine reverts acquired chemoresistance and down-regulates cancer stem cell markers in pancreatic cancer cell lines," Swiss Medical Weekly, vol. 141, Article ID w13208, 2011.

[15] L. Zhang, L. Li, M. Jiao et al., "Genistein inhibits the stemness properties of prostate cancer cells through targeting HedgehogGlil pathway," Cancer Letters, vol. 323, no. 1, pp. 48-57, 2012.

[16] A. Nanashima, G. Hatachi, T. Tsuchiya et al., "Clinical significances of cancer stem cells markers in patients with intrahepatic cholangiocarcinoma who underwent hepatectomy," Anticancer Research, vol. 33, no. 5, pp. 2107-2114, 2013.

[17] D. Yu, H.-S. Shin, Y. S. Lee, D. Lee, S. Kim, and Y. C. Lee, "Genistein attenuates cancer stem cell characteristics in gastric cancer through the downregulation of Glil," Oncology Reports, vol. 31, no. 2, pp. 673-678, 2014.

[18] H. Tanaka, M. Nakamura, and C. Kameda, “The Hedgehog signaling pathway plays an essential role in maintaining the CD44+CD24-/low subpopulation and the side population of breast cancer cells," Anticancer Research, vol. 29, no. 6, pp. 2147$2157,2009$.

[19] Y. Wakamatsu, N. Sakamoto, H. Z. Oo et al., "Expression of cancer stem cell markers ALDH1, CD44 and CD133 in primary tumor and lymph node metastasis of gastric cancer," Pathology International, vol. 62, no. 2, pp. 112-119, 2012.

[20] Y. Niu, F. Li, B. Tang, Y. Shi, Y. Hao, and P. Yu, "Clinicopathological correlation and prognostic significance of sonic hedgehog protein overexpression in human gastric cancer," International Journal of Clinical and Experimental Pathology, vol. 7, no. 8, pp. 5144-5153, 2014.

[21] Z.-S. Wang, Y. Shen, X. Li et al., "Significance and prognostic value of Gli-1 and Snail/E-cadherin expression in progressive gastric cancer," Tumor Biology, vol. 35, no. 2, pp. 1357-1363, 2014.

[22] K. Hirata, H. Suzuki, H. Imaeda et al., "CD44 variant 9 expression in primary early gastric cancer as a predictive marker for recurrence," British Journal of Cancer, vol. 109, no. 2, pp. 379386, 2013.

[23] C.-S. Yong, C.-M. Ou Yang, Y.-H. Chou, C.-S. Liao, C.-W. Lee, and C.-C. Lee, "CD44/CD24 expression in recurrent gastric cancer: a retrospective analysis," BMC Gastroenterology, vol. 12, article 95, 2012. 


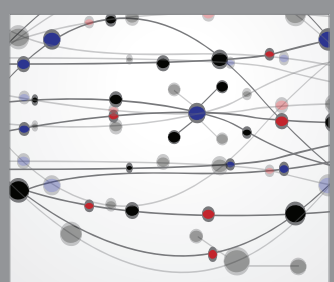

The Scientific World Journal
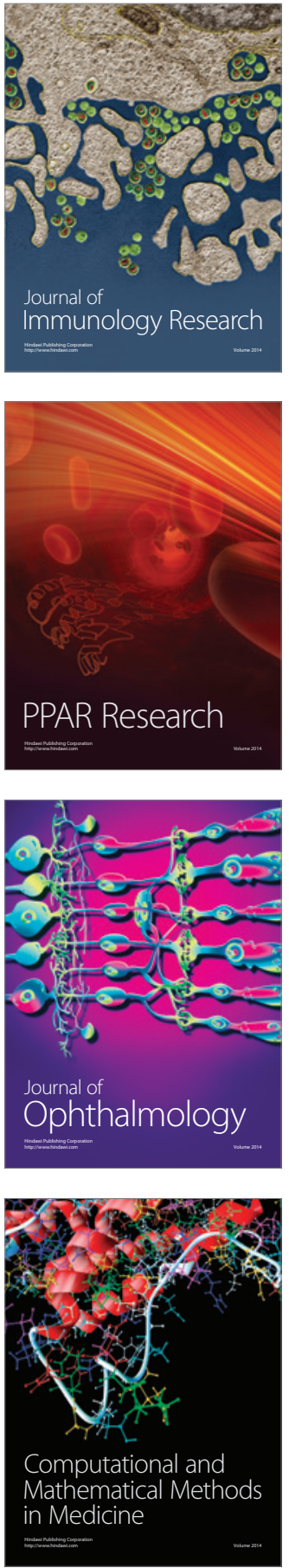

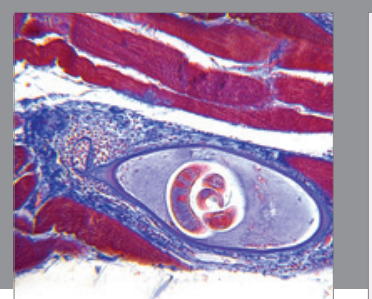

Gastroenterology Research and Practice

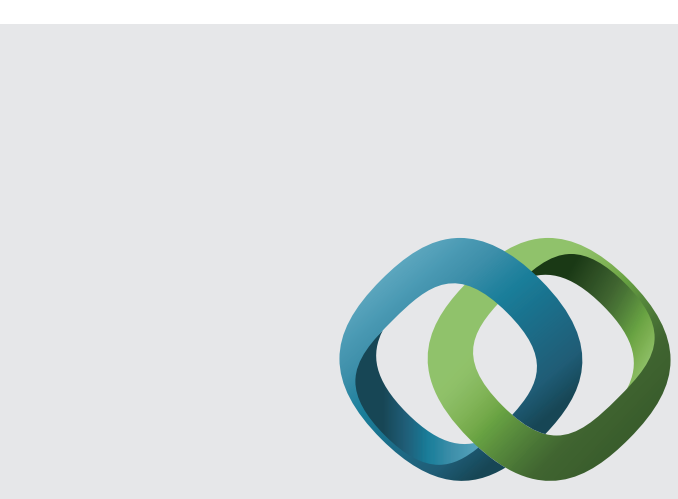

\section{Hindawi}

Submit your manuscripts at

http://www.hindawi.com
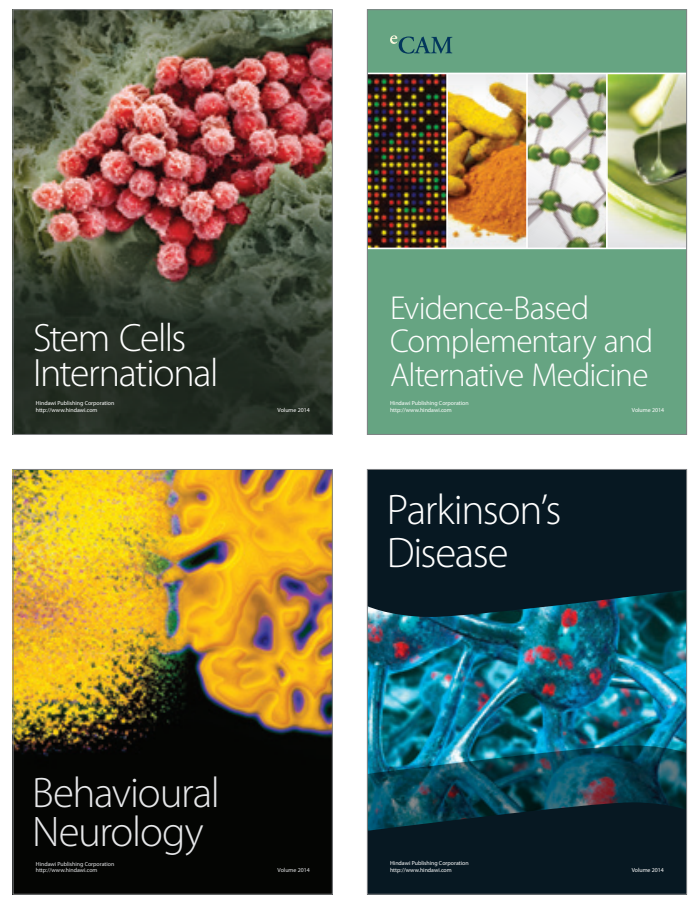
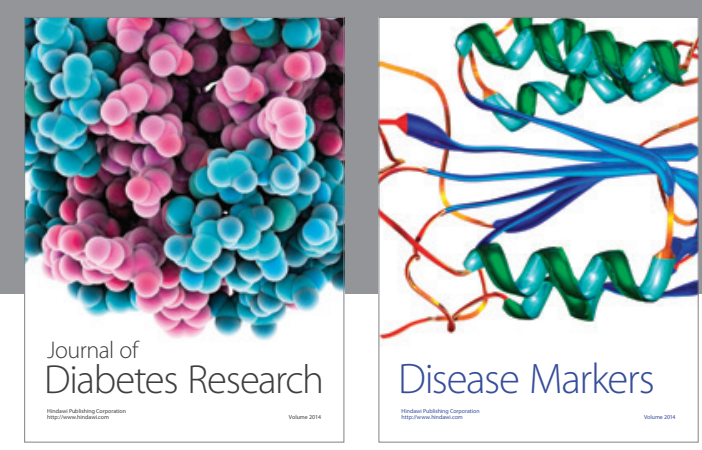

Disease Markers
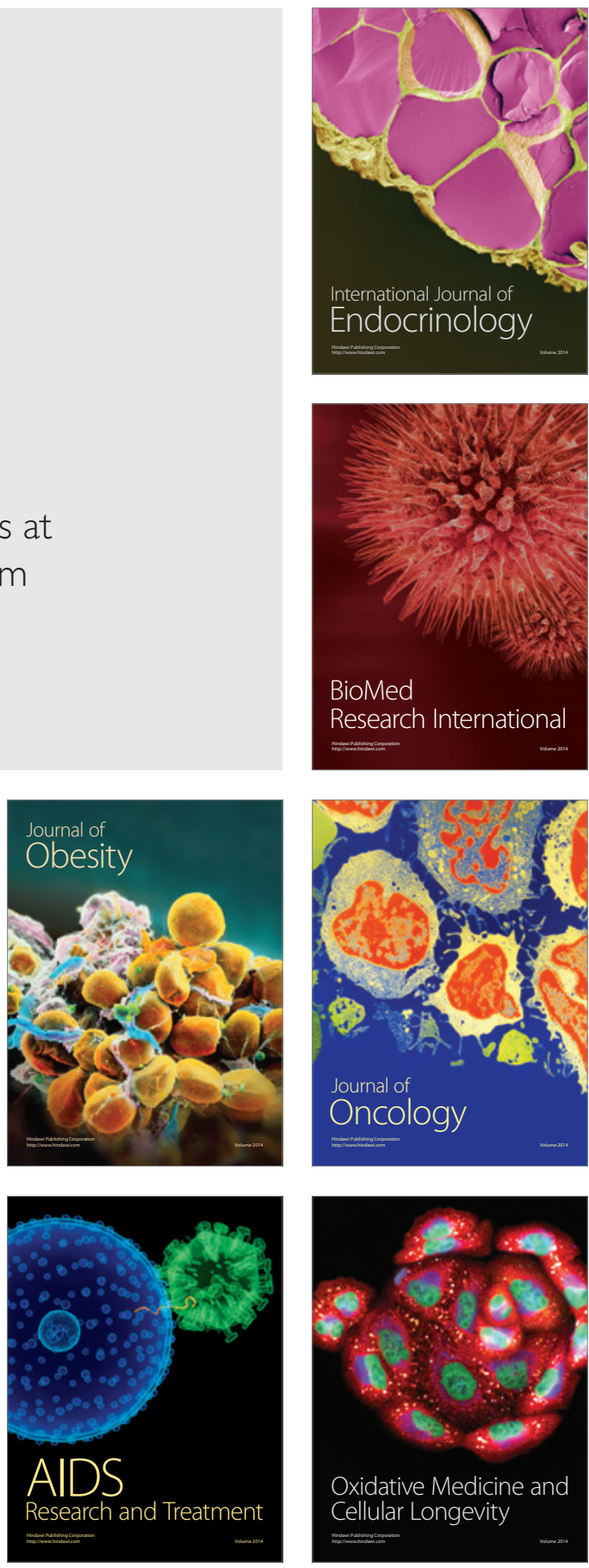